\title{
Mini-implants for direct or indirect orthodontic anchorage
}

\author{
Abstracted from \\ Chen Y, Kyung HM, Zhao WT, Yu WJ. \\ Critical factors for the success of orthodontic mini-implants: a systematic review. \\ Am J Orthod Dentofacial Orthop 2009; 135: 284-291 \\ Address for correspondence: Yan Chen, Oral Department, Attached Hospital, \\ Inner Mongolia Medical University, \#1 North Street of Tongdao, Hohhot, \\ People's Republic of China 010050. E-mail: Paifeng66@hotmail.com
}

Question: What factors effect the use of mini-implants for direct or indirect orthodontic anchorage?

Data sources Studies were sourced using Medline, the Cochrane Library and hand searches of key orthodontic journals Study selection The review was restricted to peer-review articles (randomised controlled studies, prospective clinical studies and retrospective clinical studies) dealing with mini-implants (implant diameter smaller than $2.5 \mathrm{~mm}$ ) and conducted in humans. Articles were excluded if: they dealt with standard dental implants, onplants (palatal implants), miniplates used as orthodontic anchorage, or miniscrews or microscrews for dental surgery, and implant materials research; if they were animal studies, in-vitro studies, case reports and case series, or technique presentations of mini-implants and micro-implants, review articles and letters; or if they were articles that did not meet the objective of this review or were reported in a language other than English.

Data extraction and synthesis Data were extracted independently by two authors, with disagreements resolved by discussion. Study quality was assessed. A qualitative synthesis was conducted.

Results Sixteen articles met the inclusion criteria and, because of quality limitations, the literature was summarised in two broad areas, placement-related and loading-related factors.

Conclusions Mini-implants are effective as anchorage, and their success depends on proper initial mechanical stability and loading quality and quantity.

\section{Commentary}

The use of temporary anchorage devices has become one of the routine treatment options in orthodontics. Such devices, namely miniimplants, used for orthodontic purposes can be divided into three groups: miniscrews/microscrews, miniplates, and palatal implants (onplants). A number of reviews have been published on miniimplants in the last 5 years. These reviews deal mainly with the clinical performance of miniscrews.

The study by Chen et al. addressed critical factors in the performance of miniscrews for orthodontic anchorage. The authors conclud- ed that the diameter and length of the implant should be $0.2-0.5 \mathrm{~mm}$ larger than the width and depth of the bone hole for optimal placement torque, and the selection of implant size depends on the bone available. Most of the studies included in this review are prospective ,with valid measurement methods, but none of them has a comparison group. Therefore, no conclusion could be drawn on whether there is a difference between the anchorage provided by mini-implants or traditional methods. In fact, a recent Cochrane review found only one randomised clinical trial in this area of adequate quality, which was on palatal implants. Interestingly, this trial showed no treatment changes between patients with orthodontic anchorage supported with a midpalatal implant compared with headgear. There were important differences in the movement of teeth in the groups, however, which is not surprising since headgear is known to have both skeletal and dental effects on growing subjects.

A number of clinical and biological questions still need to be answered regarding the use of mini-implants for orthodontic anchorage. Important clinical questions include the ideal locations for implant placement, anchorage loss compared with a traditional anchorage regime, stability of the anchorage devices, and treatment results and patient discomfort. Important biological questions are: the effect of the healing process and implant surface on osseo-integration (from primary stability to biological stability), inflammatory reactions such as indicated by gingival crevicular fluid biochemistry markers and periodontal microflora.

\section{Practice points}

- While temporary anchorage device in orthodontic treatment use has increased there are still important clinical and biological questions to be answered regarding the use of mini-implants for orthodontic anchorage.

Yijin Ren

Department of Orthodontics, University Medical Centre Groningen, University of Groningen, Groningen, The Netherlands

1. Skeggs RM, Benson PE, Dyer F. Reinforcement of anchorage during orthodontic brace treatment with implants or other surgical methods. Cochrane Database Syst Rev 2007 18: issue 3.

Evidence-Based Dentistry (2009) 10, 113. doi:10.1038/sj.ebd.6400687 